\title{
The origin of $s$-process isotope heterogeneity in the solar protoplanetary disk
}

\author{
Mattias EK ${ }^{1,2 *}$, Alison C. HunT ${ }^{2}$, MARIA LUGARO ${ }^{3,4}$, \\ MARIA SCHÖNBÄCHLER ${ }^{2}$ \\ ${ }^{1}$ Bristol Isotope Group, University of Bristol, UK. \\ (*mattias.ek@bristol.ac.uk). \\ ${ }^{2}$ Institute for Geochemistry and Petrology, ETH Zürich, \\ Switzerland. \\ ${ }^{3}$ Konkoly Observatory, Research Centre for Astronomy and \\ Earth Sciences, MTA Centre for Excellence, Hungary. \\ ${ }^{4}$ Monash Centre for Astrophysics, Monash University, \\ Australia.
}

Nucleosynthetic isotope variations among bulk meteorites represent the heterogenous distribution of stardust, also called presolar grains, in the protoplanetary disk. Stardust has preserved the extreme isotope composition of its stellar birth environments. We present our new model to explain both the origin of the Zr-Mo-Ru-Pd isotope correlation identified in meteorites and the missing or subdued isotopic variations in more volatile and heavy refractory elements $(Z \geq 56)[1]$.

Stardust, primarily made up of material carrying an $s$ process signature [2], accounted for a few percent of the dust inherited by our protoplanetary disk [3]. The remaining dust formed in the interstellar medium (ISM) and therefore had an isotope composition similar to the bulk Solar System (SS). We propose that thermal processing during the infall phase or in the protoplanetary disk preferentially destroyed ISM dust leading to an enrichment of stardust in regions closer to the Sun. This recreates the correlated $s$-process variation reported for different elements (e.g., Zr, Mo and Ru) in the SS.

Incomplete condensation of elements around asymptotic giant branch (AGB) stars, the site of $s$-process nucleosynthesis, led to stardust that was depleted in the less refractory elements [4]. This can account for both the smaller nucleosynthetic Pd variations [1] relative to those of the more refractory $\mathrm{Mo}$ and $\mathrm{Ru}$, and the lack of variation in more volatile elements like $\mathrm{Cd}$ [5]. The subdued or missing nucleosynthetic effects in heavy refractory elements suggest that a large fraction of stardust in the SS originated from high metallicity AGB stars as the production of heavier elements in AGB stars decreases with increasing metallicity [6]. Consequently, stardust had a lower abundance of heavy refractory elements relative to the bulk SS composition.

[1] Ek et al. (2019) Nat. Astron, in press [2] Zhukovska et al. (2008) $A \& A 479$ [3] Hoppe \& Leitner (2017) Nat. Astron. 1 [4] Ireland et al. (2018) GCA 221 [5] Toth et al. (2020) GCA, in press [6] Cseh et al. (2018) A\&A. 620 